\title{
Peritoneal Metastatic Goblet-Cell Carcinoid Tumor Treated With Cytoreductive Surgery and Intraperitoneal Chemotherapy
}

\author{
Sang Il Youn, Hwan Namgung, Jeong Seok Yun, Yun Jun Park, Dong-Guk Park \\ Department of Surgery, Dankook University School of Medicine, Cheonan, Korea
}

We report a case of a goblet-cell carcinoid tumor of the appendix which metastasized to the peritoneum and was treated by using cytoreductive surgery (CRS) with intraperitoneal chemotherapy. A 47-year-old male presented with chronic constipation and was diagnosed as having a rectal adenocarcinoma with a signet-ring-cell component under colonoscopy. Computed tomography suggested peritoneal metastases with diffuse nodular parietal peritoneal thickening of the entire abdomen and focal invasion of the upper rectum by a seeding mass. CRS with intraperitoneal chemotherapy was done under the diagnosis of a rectal adenocarcinoma with peritoneal metastases. The pathologic diagnosis was a goblet-cell carcinoid tumor of the appendix with peritoneal metastasis. The histological discrepancy between a peritoneal metastatic mass and a rectal mass was due to the mixed histological pattern of a goblet-cell carcinoid tumor. A metastatic mass may not share identical immunohistochemical characteristics from its origin. This histologic discrepancy necessitates caution in diagnosing a distant metastasis of a goblet-cell carcinoid tumor.

\section{Keywords: Appendix; Carcinoid tumor; Goblet cells; Peritoneal neoplasms; Intraperitoneal infusion}

\section{INTRODUCTION}

Goblet-cell carcinoid (GCC) tumors are rare tumors arising almost exclusively in the appendix [1] and having a mixed neuroendocrine and goblet-cell-type morphology [2]. A GCC was first recognized as a separate entity in 1969 by Gagne et al. [3]. We report a GCC of the appendix which manifested as metastasis to the peritoneum. This case shows diverse histological findings between the primary site lesion and the metastatic lesion.

\section{CASE REPORT}

A 47-year-old male visited a local hospital presenting with no

Received: November 25, 2014 - Accepted: December 22, 2014

Correspondence to: Dong-Guk Park, M.D.

Department of Surgery, Dankook University School of Medicine,

119 Dandae-ro, Dongnam-gu, Cheonan 330-997, Korea

Tel: +82-41-550-3931, Fax: +82-41-565-6167

E-mail:dkpark@dankook.ac.kr

(C) 2015 The Korean Society of Coloproctology

This is an open-access article distributed under the terms of the Creative Commons Attribution NonCommercial License (http://creativecommons.org/licenses/by-nc/3.0) which permits unrestricted noncommercial use, distribution, and reproduction in any medium, provided the original work is properly cited. other symptoms except for several months of chronic constipation. A colonoscopic exam revealed a mass-like lesion in the rectum, and pathology confirmed the lesion to be an adenocarcinoma with a signet-ring-cell carcinoma component (Fig. 1). Full examination of the entire colon failed due to the patient's complaining of severe pain as the scope passed through the cecal area. A subsequent computed tomography scan showed a peritoneal carcinomatosisascites along the upper abdomen, diffuse nodular parietal peritoneal thickening along the perihepatic space extending into the perisplenic area and the right lateral abdomen, a diffuse peritoneal seeding nodular mass lesion along the mesentery of the right mid to lower abdomen, central the mid to lower abdomen, a rectovesical space, suggestive of focal invasion of urinary bladder's posterior and superior wall side, and focal invasion of the upper rectum by the seeding mass lesion (Fig. 2).

The initial impression was rectal cancer with peritoneal seeding. Upon surgical examination, extensive metastatic seeding nodules were seen throughout the abdominal cavity, including the entire peritoneum, as well as the colon, small bowel mesentery, surface of the stomach, spleen, diaphragm, and surface of the liver (Fig. $3 \mathrm{~A})$. The most severe metastatic region was the pelvic cavity. The rectum, distal ileum, and metastatic masses were conglomerated to form a hard mass. The rectal mass, which was initially thought 

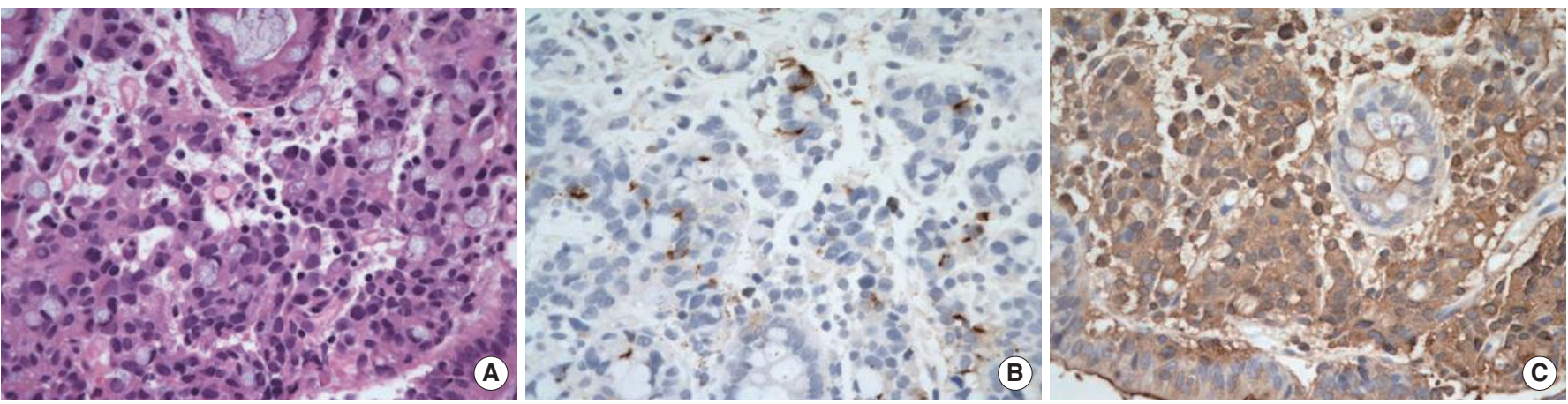

Fig. 1. Microscopic and immunohistochemical findings of the rectum (previous biopsy): (A) adenocarcinoma, poorly differentiated, with a signet-ring-cell carcinoma component on H\&E staining $(\times 400)$, (B) very focally positive chromogranin A $(\times 400)$, and $(\mathrm{C})$ diffusely positive carcinoembryonic antigen, negative synaptophysin (CD56) and about an 80\% Ki-67-labeling index $(\times 400)$.
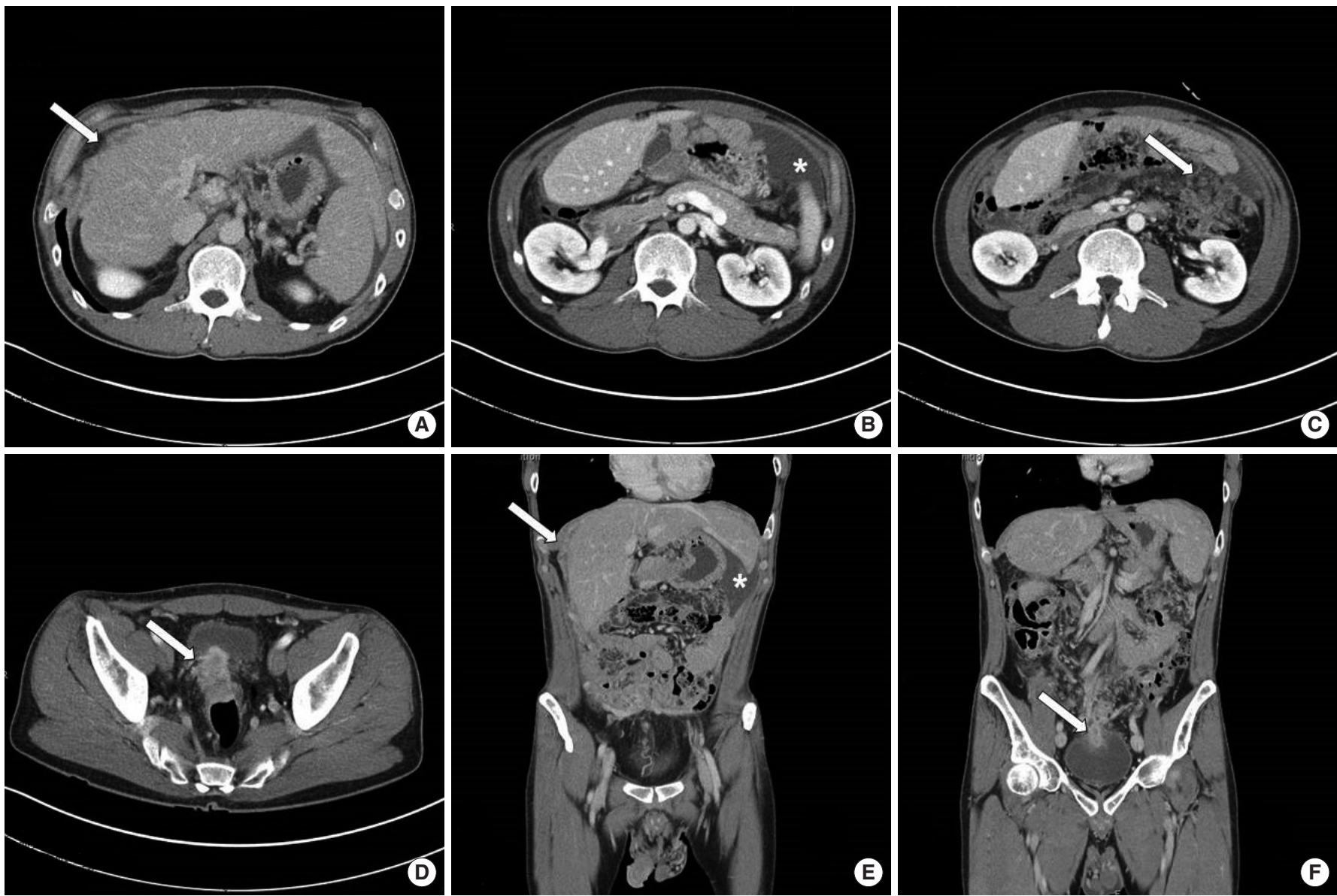

Fig. 2. The findings of abdomino-pelvic computed tomography: peritoneal carcinomatosis, ascites along the upper abdomen (B, E, asterisk), diffuse nodular parietal peritoneal thickening along the perihepatic space extending into the perisplenic area and the right lateral abdomen (A, C, E, white arrow), a diffuse peritoneal seeding nodular mass lesion along the mesentery of the right mid to lower abdomen, cenetral mid to lower abdomen (C, white arrow), rectovesical space, suggestive of focal invasion of the urinary bladder posterior and superior wall side, focal invasion of the upper rectum by a seeding mass lesion (D, F, white arrow).

to be a primary mass, proved to be a metastatic mass penetrating the rectum. A peritonectomy, omentectomy, total colectomy, sple- nectomy, small bowel resection, and end ileostomy were performed (Fig. 3B), and early postoperative intraperitoneal chemo- 

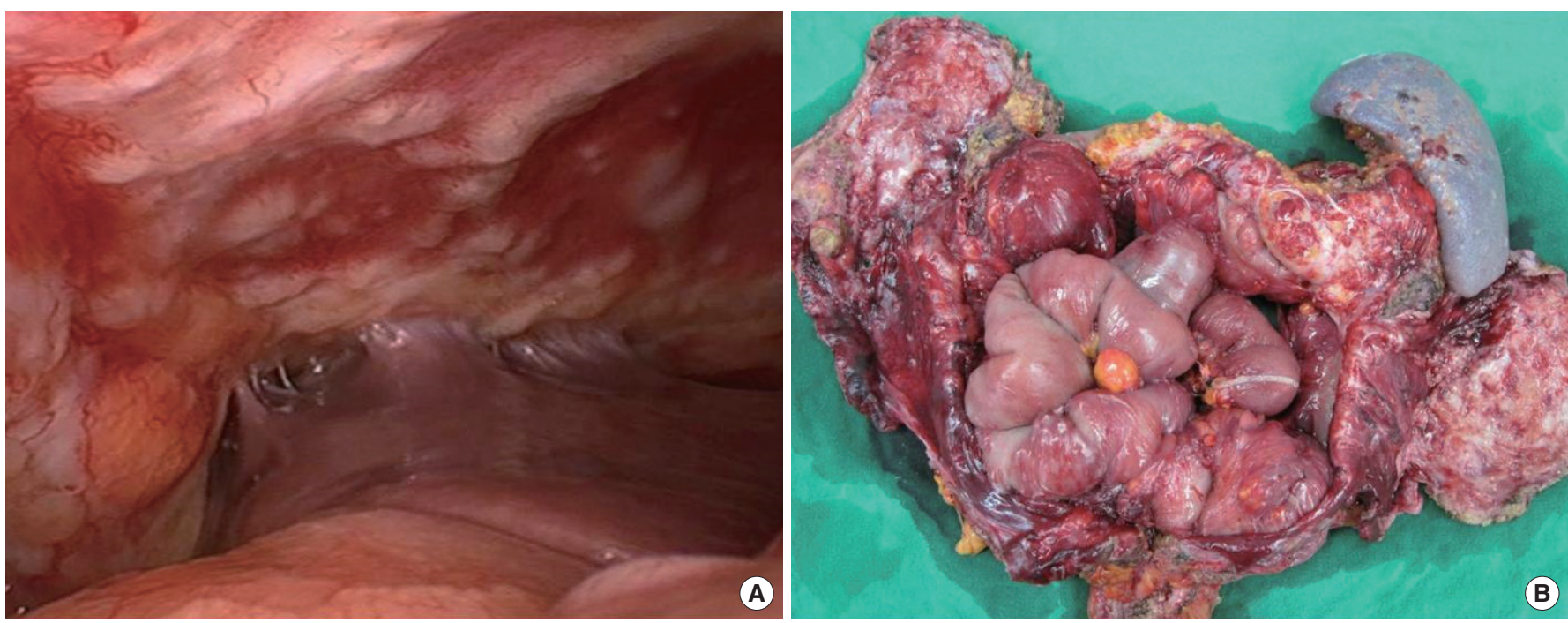

Fig. 3. (A) Laparoscopic finding of peritoneal metastases of a goblet-cell carcinoid, and (B) en bloc resected specimen: total peritonectomy, total colectomy with long distal ileal segment and splenectomy.

therapy was done for 5 days. The first day we used $20 \mathrm{mg}$ of mitomycin C in 23 hours. After drainage, 5-fluorouracil (5-FU) (900 $\mathrm{mg}, 15 \mathrm{mg} / \mathrm{kg}$ ) in $1.5 \mathrm{~L}$ of warmed peritoneal dialysate was introduced into the abdominal cavity for 23 hours for another 4 days.

The pathologic diagnosis was a GCC $(8 \mathrm{~cm} \times 8 \mathrm{~cm} \times 7 \mathrm{~cm})$ arising from the appendix. The mass had penetrated the visceral peritoneum with tumor metastases to 4 of 9 regional lymph nodes. The immunohistochemical result of the GCC showed positive for chromogranin A, synaptophysin, carcinoembryonic antigen, and CK20. The Ki-67 was moderate to high in proliferative activity. Meanwhile, the rectal mass showed features of a poorly differentiated adenocarcinoma with a signet-ring-cell component. No components of chromogranin A, synaptophysin, or CD56 were found in the metastatic mass (Fig. 4).

A 5-day regimen of intraperitoneal chemotherapy was administered, after which the patient was discharged. Subsequent to his discharge, the patient underwent 5-FU+Oxaliplatin (FOLFOX4) chemotherapy. The patient remains well without any recurrence or any complications at 6 months after the surgery.

\section{DISCUSSION}

GCC tumors are unusual tumors of the appendix and were first described by Subbuswamy et al. [4] in 1974. While an appendiceal adenocarcinoma mimics the characteristics of a colonic adenocarcinoma and an appendiceal carcinoid tumor shows a neuroendocrine phenotype, an appendiceal GCC shows a mixed phenotype of neuroendocrine differentiation and intestinal-type goblet-cell morphology [5]. So far, they are thought to originate from pluripotent intestinal crypt-base stem cells [6, 7]. Immunohistologically, GCCs are positive for chromogranin A and synaptophysin. They are crucial biomarkers for classic neuroendocrine tumors, showing diffuse positivity in carcinoid tumors. In a GCC, however, only the focal parts of the neoplasm are stained positive. This may mean that the biopsy from the metastatic mass may lack cell components that stain positive for chromogranin A or synaptophysin. As a result, the immunoprofile of the metastatic tumor can vary from that of its primary tumor. Our case shows that this discrepancy in the immunohistochemical results may be a confounding factor in confirming the diagnosis of the primary tumor.

Because a GCC tumor does not present with any specific symptoms other than an acute abdomen, diagnosis is almost always made postoperatively and often in a late stage of the disease. One review study showed that $11.16 \%$ of the patients presented with a distant metastasis at the time of diagnosis, ovaries being the most common with an incidence of $3.60 \%$, followed by abdominal carcinomatosis with an incidence of $1.03 \%$ [7]. In our case, the peritoneal seeding disseminated diffusely into the entire abdomen. The seeding nodule in the rectum had infiltrated the bowel wall from the serosal side, causing the metastatic mass to be mistaken for a rectal mass. Despite its low prevalence, a GCC should be a consideration when multiple tumors are found in the appendix and colon. Also, a full examination of the gastrointestinal tract needs to be done before deciding on the primary focus of the tumor.

The treatment for a GCC is surgical removal of the tumor, and a GCC can usually be successfully treated with a simple appendectomy. Controversies exist about whether a right hemicolectomy should be performed or a simple appendectomy would suffice. In a review of surgeries performed on patients with a GCC, a simple appendectomy was performed in $24.57 \%$ of the cases and right hemicolectomy in $34.7 \%$ of the cases [7]. The treatment modality for a GCC with peritoneal seeding is also controversial, but recent 

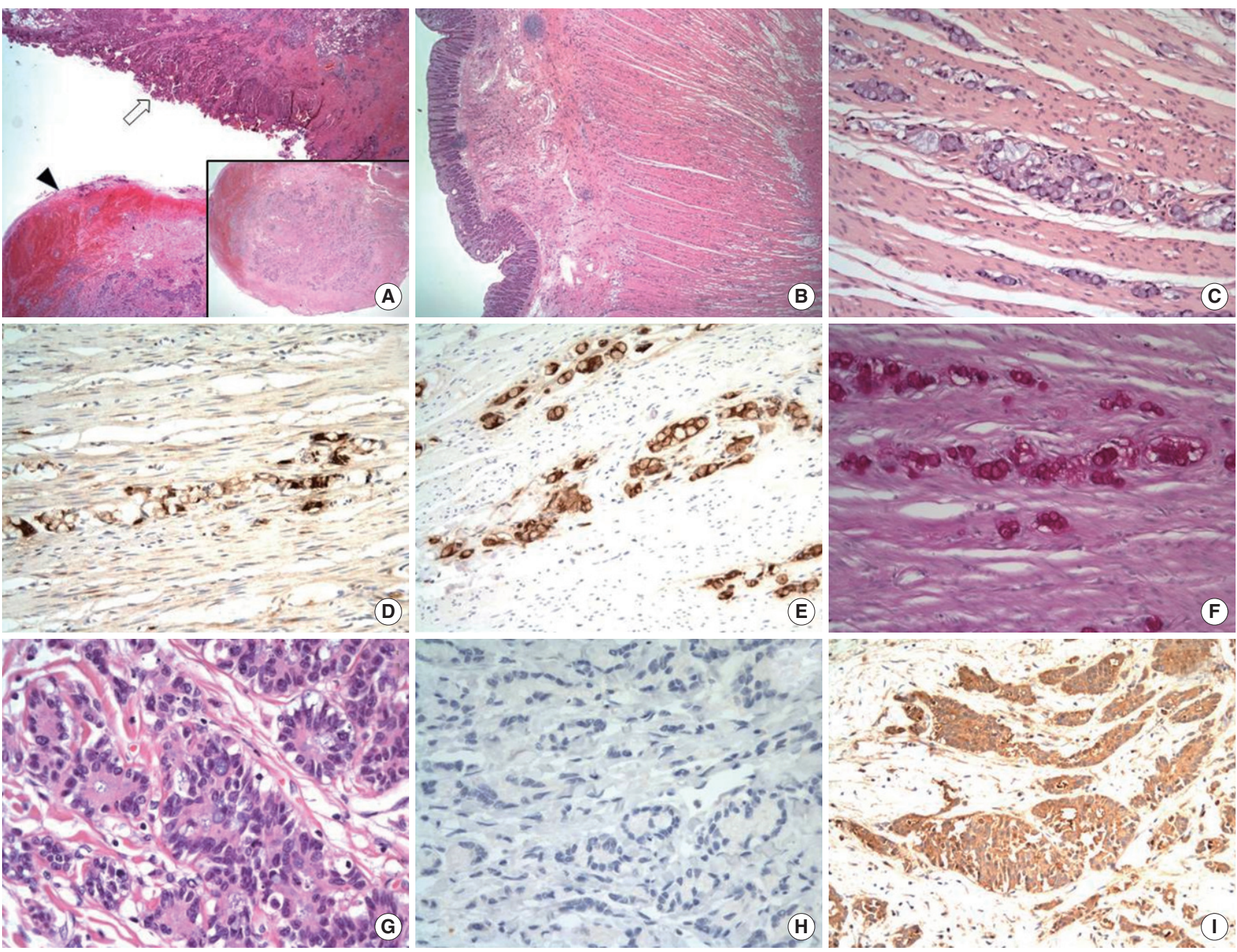

Fig. 4. Microscopic and immunohistochemical findings of the appendic and peritoneal metastatic mass: (A) peritoneum with tumor cell infiltration (arrow) and concentric infiltration of tumor cells through the appendiceal wall (arrowhead and inlet) $(\times 100)$, and (B) mural infiltration of tumor cells with preservation of the mucosa of the colon $(\times 100)$. (C) H\&E staining of the peritoneal metastatic mass $(\times 200)$, and $(\mathrm{D})$ chromogranin A, a neuroendocrine marker $(\times 200)$, is positive, (E) negative carcinoembryonic antigen (CEA), an adenocarcinoma marker $(\times 200)$, and $(F)$ positive diastase resistant periodic acid-Schiff, mucin staining $(\times 400)$. The predominant tumor cells were goblet-cell carcinoid cells in panels C-F. (G) H\&E staining on another lesion showed the tumor cells to be a typical adenocarcinoma $(\times 400)$, and $(\mathrm{H})$ negative chromogranin $(\times 200)$, as well as (I) positive CEA, staining $(\times 200)$. The predominant tumor cells were goblet-cell carcinoid cells, and a minor tumor component was a typical adenocarcinoma.

reports advocate cytoreductive surgery (CRS) followed by intraperitoneal chemotherapy [8-11]. Our patient initially showed a peritoneal cancer index (PCI) of 30, and he showed completeness of cytoreduction of CC2. In appendiceal tumors, the PCI is not important as a prognostic factor [12]. Because appendiceal tumors show good response to chemotherapy and the patient was fairly young and apparently healthy, we proceeded with the surgery. Evidently, complete cytoreduction is required for optimal results, but in our case, the tumor dissemination was too advanced.
Although a complete resection of the tumor cells was not possible in our case, aggressive debulking surgery combined with intraperitoneal and systemic chemotherapy proved to be a successful option for the treatment of the advanced appendiceal GCC with peritoneal seeding. However, further clinical follow up and an accumulation of cases are needed to obtain more knowledge concerning the prognosis for patients presenting with a GCC with peritoneal seeding. 


\section{CONFLICT OF INTEREST}

No potential conflict of interest relevant to this article was reported.

\section{REFERENCES}

1. Gui X, Qin L, Gao ZH, Falck V, Harpaz N. Goblet cell carcinoids at extraappendiceal locations of gastrointestinal tract: an underrecognized diagnostic pitfall. J Surg Oncol 2011;103:790-5.

2. Abt AB, Carter SL. Goblet cell carcinoid of the appendix. An ultrastructural and histochemical study. Arch Pathol Lab Med 1976; 100:301-6.

3. Gagne F, Fortin P, Dufour V, Delage C. Tumors of the appendix associating histologic features of carcinoid and adenocarcinoma. Ann Anat Pathol (Paris) 1969;14:393-406.

4. Subbuswamy SG, Gibbs NM, Ross CF, Morson BC. Goblet cell carcinoid of the appendix. Cancer 1974;34:338-44.

5. Carr NJ, McCarthy WF, Sobin LH. Epithelial noncarcinoid tumors and tumor-like lesions of the appendix. A clinicopathologic study of 184 patients with a multivariate analysis of prognostic factors. Cancer 1995;75:757-68.

6. Tang LH, Shia J, Soslow RA, Dhall D, Wong WD, O’Reilly E, et al.
Pathologic classification and clinical behavior of the spectrum of goblet cell carcinoid tumors of the appendix. Am J Surg Pathol 2008;32:1429-43.

7. Pahlavan PS, Kanthan R. Goblet cell carcinoid of the appendix. World J Surg Oncol 2005;3:36.

8. Pham TH, Wolff B, Abraham SC, Drelichman E. Surgical and chemotherapy treatment outcomes of goblet cell carcinoid: a tertiary cancer center experience. Ann Surg Oncol 2006;13:370-6.

9. Varisco B, McAlvin B, Dias J, Franga D. Adenocarcinoid of the appendix: is right hemicolectomy necessary? A meta-analysis of retrospective chart reviews. Am Surg 2004;70:593-9.

10. Mahteme H, Sugarbaker PH. Treatment of peritoneal carcinomatosis from adenocarcinoid of appendiceal origin. Br J Surg 2004; 91:1168-73.

11. Garin L, Corbinais S, Boucher E, Blanchot J, Le Guilcher P, Raoul JL. Adenocarcinoid of the appendix vermiformis: complete and persistent remission after chemotherapy (folfox) of a metastatic case. Dig Dis Sci 2002;47:2760-2.

12. Sugarbaker PH, Jablonski KA. Prognostic features of 51 colorectal and 130 appendiceal cancer patients with peritoneal carcinomatosis treated by cytoreductive surgery and intraperitoneal chemotherapy. Ann Surg 1995;221:124-32. 